\title{
Ethernet Technologies IEEE 802.3 Performance over VoIP Network Using OPNET Simulator
}

\author{
Salah El-din El-Hafiz Farah ${ }^{1}$, Dr. Amin Babiker A/Nabi Mustafa ${ }^{2}$ \\ ${ }^{1}$ Department of Communication Engineering, Al-Neelain University, Khartoum, Sudan
}

\begin{abstract}
The objective of the paper is to compare and evaluate the performance of different types of Ethernet technologies IEEE 802.3; using OPNET 17.5 as a simulation tool to compare them in terms of different criteria., designed a local area networks based on Ethernet technologies categories, a network simulator platform. Simulation techniques allow us to analyze the behavior of each network separately. The network consists of different components such as servers, switches, and work station as end points. In the experimental process we take output of packet received traffic by a server, delay and jitter, in VOIP environment. The main Objective of this research is to find out what is the best Ethernet technologies speed can be used to a real time application (VOIP) that can be effect with any type of delay exclude the delay of transmission line finally Understanding the impact of the data link transmission delay, jitter and voice traffic received by application server. The simulation has been carried out using Optimized Network Engineering Tool (OPNET modeler academic edition 17.5).
\end{abstract}

Keywords: Ethernet, Delay, Jitter, OPNET, VOIP, Traffic received, IEEE

\section{Introduction}

\subsection{Background}

Ethernet is a family of technologies that provides data-link and physical specifications for controlling access to a shared network medium. It has emerged as the dominant technology used in LAN networking.

Ethernet was originally developed by Xerox in the 1970s, and operated at $2.94 \mathrm{Mbps}$. The technology was standardized as Ethernet Version 1 by a consortium of three companies DEC, Intel, and Xerox, collectively referred to as DIX - and further refined as Ethernet II in 1982.

In the mid-1980s, the Institute of Electrical and Electronic Engineers (IEEE) published a formal standard for Ethernet, defined as the IEEE 802.3 standard. The original 802.3 Ethernet operated at $10 \mathrm{Mbps}$, and successfully supplanted competing LAN technologies, such as Token Ring.

Ethernet has several benefits over other LAN technologies:

- Simple to install and manage

- Inexpensive

- Flexible and scalable

- Easy to interoperate between vendors.

\subsection{IEEE standards}

IEEE developed a set of network standards. They include

1)IEEE 802.1: Standards related to network management.

2)IEEE 802.2: General standard for the data link layer in the OSI Reference Model. The IEEE divides this layer into two sub-layers -- the logical link control (LLC) layer and the media access control (MAC) layer. The MAC layer varies for different network types and is defined by standards IEEE 802.3 through IEEE 802.5

3)IEEE 802.3: Defines the MAC layer for bus networks that use CSMA/CD. This is the basis of the Ethernet standard.
4)IEEE 802.4: Defines the MAC layer for bus networks that use a token-passing mechanism (token bus networks).

5)IEEE 802.5: Defines the MAC layer for token-ring networks.

6)IEEE 802.6: Standard for Metropolitan Area Networks (MANs).

\subsection{Ethernet Cabling Types:}

Ethernet can be deployed over three types of cabling:

- Coaxial cabling - almost entirely deprecated in Ethernet networking

- Twisted-pair cabling

- Fiber optic cabling

Coaxial cable, often abbreviated as coax, consists of a single wire surrounded by insulation, a metallic shield, and a plastic sheath. The shield helps protect against electromagnetic interference (EMI), which can cause attenuation, a reduction of the strength and quality of a signal. EMI can be generated by a variety of sources, such as florescent light ballasts, microwaves, cell phones, and radio transmitters.

(Coax is commonly used to deploy cable television to homes and businesses.)

\subsection{Categories of Ethernet}

The original 802.3 Ethernet standard has evolved over time, supporting faster transmission rates, longer distances, and newer hardware technologies. These revisions or amendments are identified by the letter appended to the standard, such as $802.3 \mathrm{u}$ or $802.3 \mathrm{z}$. Major categories of Ethernet have also been organized by their speed:

- Ethernet (10Mbps)

- Fast Ethernet (100Mbps)

- Gigabit Ethernet

- 10 Gigabit Ethernet 


\section{International Journal of Science and Research (IJSR) \\ ISSN (Online): 2319-7064}

Index Copernicus Value (2013): 6.14 | Impact Factor (2014): 5.611

\section{A.Ethernet (10 Mbps)}

Ethernet is now a somewhat generic term, describing the entire family of technologies. However, Ethernet traditionally referred to the original 802.3 standard, which operated at 10 Mbps. Ethernet supports coax, twisted-pair, and fiber cabling. Ethernet over twisted-pair uses two of the four pairs.

\section{B. Fast Ethernet (100 Mbps):}

In 1995, the IEEE formalized $802.3 \mathrm{u}$, a $100 \mathrm{Mbps}$ revision of Ethernet that became known as Fast Ethernet. Fast Ethernet supports both twisted-pair copper and fiber cabling, and supports both half-duplex and full-duplex.

\section{C.Gigabit Ethernet:}

Gigabit Ethernet operates at $1000 \mathrm{Mbps}$, and supports both twisted-pair (802.3ab) and fiber cabling (802.3z). Gigabit over twisted-pair uses all four pairs, and requires Category $5 \mathrm{e}$ cable for reliable performance.

\section{Methodology}

The network is implemented by using different network after the network implementation; start to configure the attributes for different Ethernet connection, three parameters (Ethernet delay, jitter and traffic received by server (bit per sec)) has considered to evaluate the network performance for each VOIP transmission method.

\section{Network Scenario Components}

The components used in the network models running on OPNET 17.5 device used in the network are 30 work stations, one Ethernet switch (32 port), and one application server work as a call manager express. To represent and supporting Voice transaction between workstations and switch, the switch (Ethernet 32) are used. The IP packets arriving on the input interface are switched to the appropriate output interface based on packet destination IP address. The setup uses the following models and configurations and for the purpose described below:

- Ethernet 32 port_switch node: This node is used to represent a switch supporting up to 32 Ethernet interfaces. Ethernet Work stations node this node is used to represent a workstation with client-server applications running over TCP/IP and UDP/IP.

- Application Configuration: This table lists various parameters and their descriptions for the different applications (i.e. web browser HTTP Heavy and FTP heavy applications). The specified application name is used while creating user profiles on the "Profile Configuration" object.

- Profile Configuration: This is used to create user profiles. These user profiles can be specified on different nodes in the network to generate application layer traffic. The applications defined in the Application Configuration are used by this object to configure profiles. Traffic patterns can be specified followed by the configured profiles and the applications.

- Ethernet server model: The Ethernet server model represents a server node with server applications running over TCP/IP and UDP/IP. This node supports one underlying Ethernet connection at $10 \mathrm{Mbps}, 100 \mathrm{Mbps}$, or 1 Gbps. The operational speed is determined by the connected link's data rate.

- Ethernet workstation: The Ethernet work station node model represents a workstation with client-server applications running over TCP/IP and UDP/IP. The workstation supports one underlying Ethernet connection at $10 \mathrm{Mbps}, 100 \mathrm{Mbps}$, or $1000 \mathrm{Mbps}$.

This workstation requires a fixed amount of time to route each packet, as determined by the "IP Forwarding Rate" attribute of the node. Packets are routed on a first-come-firstserve basis and may encounter queuing at the lower protocol layers, depending on the transmission rates of the corresponding output interfaces.

\section{Network Architecture}

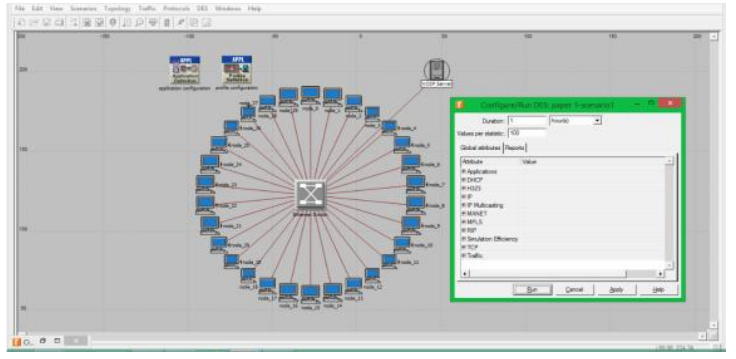

Figure 1: Network topology implemented Scenario

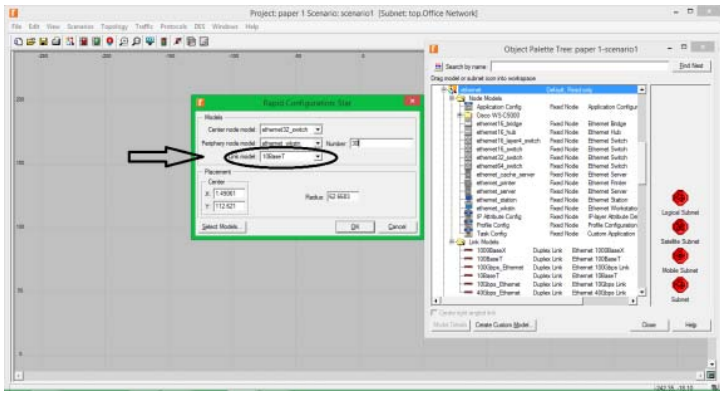

Figure 2: Type of Ethernet 10 BASE T

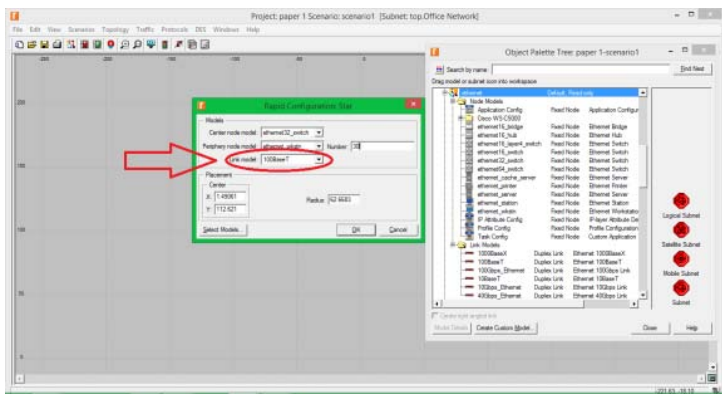

Figure 3: Type of Ethernet 100 BASE T

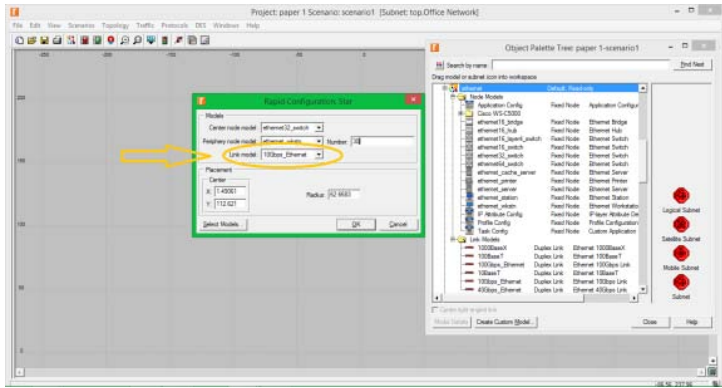

Figure 4: Type of Ethernet $10 \mathrm{Gbps}$

\section{Volume 4 Issue 12, December 2015}




\section{International Journal of Science and Research (IJSR) \\ ISSN (Online): 2319-7064}

Index Copernicus Value (2013): 6.14 | Impact Factor (2014): 5.611

\section{OPNET Modeler}

The Optimized Network Engineering Tools (OPNET) Modeler is an efficient way to provide a complete study for the network analysis. The graphical user interface (GUI) is simple to use and the result is shown as graphical and static. Furthermore, it does not require a programming knowledge, and this can be easily used. The OPNET analyses the network as a real life network which gives a complete view before building the network in real life. The OPNET contains a library of protocols and models which can be used as examples.

\section{Results and Discussion}

The simulation ran for 1 hour $(3600 \mathrm{sec})$, sufficient to gain an overview of the network's behavior. The results of the three network scenarios are shown in Fig. 5 - Fig 6 - Fig. 7; 10 BASE T represented in blue, 100 BASE T represented in red and $10 \mathrm{Gbps}$ represented in green

A.Ethernet Delay in the Figure 5 below shows the comparison of delay The 10 Base $\mathrm{T}$ has a higher Delay than 100 Base $\mathrm{T}$ and 10 Gbps because 10 Base $\mathrm{T}$ has a smaller data rate speed more than the VoIP application need to establish. But other Ethernet speed less delay and make the connection with acceptable delay value.

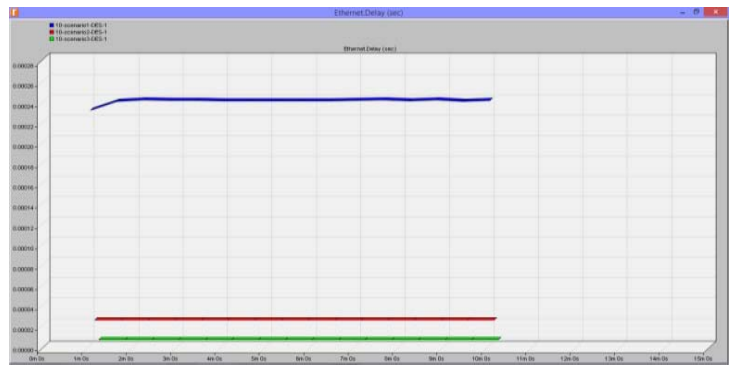

Figure 5: Ethernet delay

B. If two consecutive packets leave the source node with time stamps $\mathrm{t} 1 \& \mathrm{t} 2$ and are played back at the destination node at time $\mathrm{t} 3 \& \mathrm{t} 4$, then:

$$
\text { Jitter }=(t 4-t 3)-(t 2-t 1(
$$

Negative jitter indicates that the time difference between the packets at the destination node was less than that at the source node. Voice jitter in the Figure 6 below shows the comparison of jitter the 10 Base $\mathrm{T}$ had a frequently value of jitter and the other its more stable jitter value .

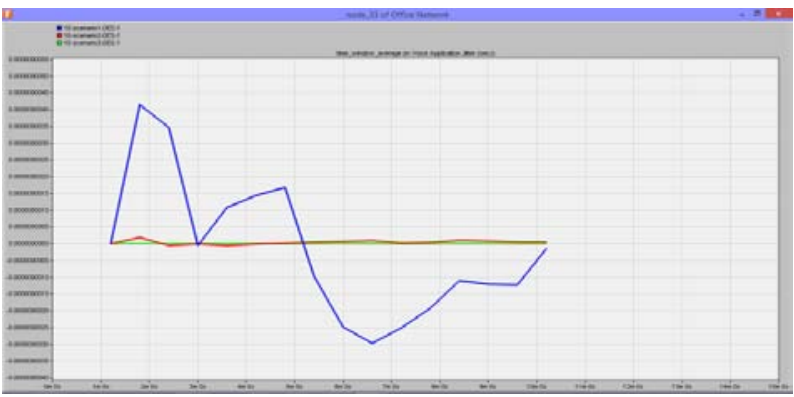

Figure 6: voice jitter
C. Traffic received by server Figure 7 below shows the comparison of Voice traffic received (bits/sec) and shows three values of theoretical have equal state and stable because the traffic generated by simulator does not change. Also the small change of values dose not affected quality of service in this case.

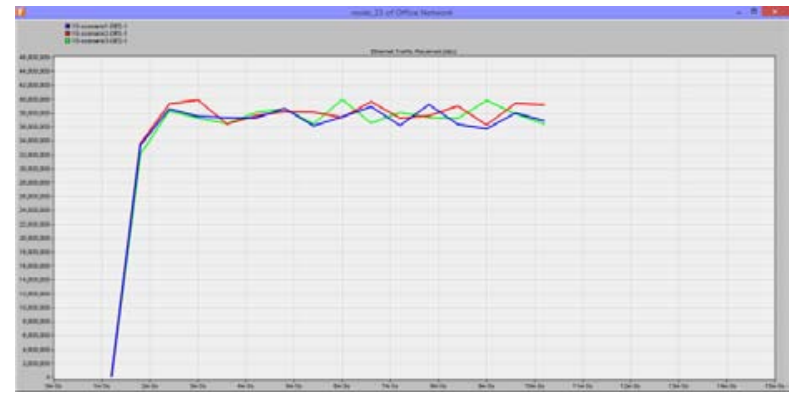

Figure 7: Traffic received by server

\section{Conclusion}

We have evaluated the delay, jitter and traffic received by server on simulation and analytical methods in the network topology was configured in three scenarios as 10 Base T, 100 Base $\mathrm{T}$ and $10 \mathrm{Gbps}$ The statistical analysis was done to provide suitable results and to show that the network's performance varied International the throughputs of the three network simulations were analyzed by using byte

\section{Future work}

Further research is being carried out on the same direction on coaxial and fiber optics Networks.

\section{References}

[1] http://docwiki.cisco.com/wiki/Ethernet_Technologies; http://www.techfest.com/networking/lan/ethernet1.htm

[2] aaron@routeralley.com

[3] http://www.techfest.com/networking/lan/ethernet3.htm

[4] http://www.infocellar.com/networks/ethernet/frame.htm 